\title{
Aortic microcalcification is associated with elastin fragmentation in Marfan syndrome
}

\author{
Shaynah Wanga ${ }^{1,2,3 \dagger}$ (1) Stijntje Hibender ${ }^{\dagger}$, Yanto Ridwan ${ }^{4}$, Cindy van Roomen', Mariska Vos', \\ Ingeborg van der Made ${ }^{5}$, Nicole van Vliet', Romy Franken³, Luigi AMJG van Riel', Maarten Groenink3,6, \\ Aeilko H Zwinderman7, Barbara JM Mulder', Carlie JM de Vries', Jeroen Essers ${ }^{4,8}$ and Vivian de Waard'* \\ Department of Medical Biochemistry, Academic Medical Centre Amsterdam, Amsterdam, The Netherlands \\ 2 Netherlands Heart Institute, Utrecht, The Netherlands \\ Department of Cardiology, Academic Medical Centre Amsterdam, Amsterdam, The Netherlands \\ 4 Department of Molecular Genetics, Erasmus MC, Rotterdam, The Netherlands \\ 5 Department of Experimental Cardiology, Academic Medical Centre Amsterdam, Amsterdam, The Netherlands \\ 6 Department of Radiology, Academic Medical Centre Amsterdam, Amsterdam, The Netherlands \\ 7 Department of Clinical Epidemiology and Biostatistics, Academic Medical Centre Amsterdam, Amsterdam, The Netherlands \\ 8 Department of Radiation Oncology, Department of Vascular Surgery, Erasmus MC, Rotterdam, The Netherlands
}

*Correspondence to: V de Waard, Academic Medical Centre Amsterdam, Department of Medical Biochemistry, KI-I I 4, Meibergdreef I 5, I I 05 AZ Amsterdam, The Netherlands. E-mail: v.dewaard@amc.uva.nl

†Equal contributions.

\begin{abstract}
Marfan syndrome (MFS) is a connective tissue disorder in which aortic rupture is the major cause of death. MFS patients with an aortic diameter below the advised limit for prophylactic surgery $(<5 \mathrm{~cm})$ may unexpectedly experience an aortic dissection or rupture, despite yearly monitoring. Hence, there is a clear need for improved prognostic markers to predict such aortic events. We hypothesize that elastin fragments play a causal role in aortic calcification in MFS, and that microcalcification serves as a marker for aortic disease severity. To address this hypothesis, we analysed MFS patient and mouse aortas. MFS patient aortic tissue showed enhanced microcalcification in areas with extensive elastic lamina fragmentation in the media. A causal relationship between medial injury and microcalcification was revealed by studies in vascular smooth muscle cells (SMCs); elastin peptides were shown to increase the activity of the calcification marker alkaline phosphatase (ALP) and reduce the expression of the calcification inhibitor matrix GLA protein in human SMCs. In murine Fbn $7^{\mathrm{C} 10396 /+}$ MFS aortic SMCs, Alpl mRNA and activity were upregulated as compared with wild-type SMCs. The elastin peptide-induced ALP activity was prevented by incubation with lactose or a neuraminidase inhibitor, which inhibit the elastin receptor complex, and a mitogen-activated protein kinase kinase-1/2 inhibitor, indicating downstream involvement of extracellular signal-regulated kinase-1/2 (ERK1/2) phosphorylation. Histological analyses in MFS mice revealed macrocalcification in the aortic root, whereas the ascending aorta contained microcalcification, as identified with the near-infrared fluorescent bisphosphonate probe OsteoSense- 800 . Significantly, microcalcification correlated strongly with aortic diameter, distensibility, elastin breaks, and phosphorylated ERK1/2. In conclusion, microcalcification co-localizes with aortic elastin degradation in MFS aortas of humans and mice, where elastin-derived peptides induce a calcification process in SMCs via the elastin receptor complex and ERK1/2 activation. We propose microcalcification as a novel imaging marker to monitor local elastin degradation and thus predict aortic events in MFS patients.

Copyright (C) 2017 Pathological Society of Great Britain and Ireland. Published by John Wiley \& Sons, Ltd.
\end{abstract}

Keywords: Marfan syndrome; vascular calcification; aneurysm; transgenic mice

Received 26 November 2016; Revised 17 June 2017; Accepted 6 July 2017

No conflicts of interest were declared.

\section{Introduction}

Marfan syndrome (MFS) is a connective tissue disorder caused by a mutation in the fibrillin-1 gene (FBNI) [1]. This protein is a component of microfibrils in the extracellular matrix, and is essential for elastic fibre integrity [2]. Fibrillin-1 protein deficiency or mutation leads to elastin fragmentation in the aorta, contributing to the life-threatening feature of aortic aneurysm formation and dissection, as experienced by MFS patients. Aorta pathology mostly occurs in the aortic root and thoracic aorta $[3,4]$. To prevent aortic growth and dissection, MFS patients are treated with blood pressure-lowering drugs and by prophylactic aortic (root) replacement [5]. 
The timing of surgery is based on several risk factors, including aortic diameter, the site of dilatation, the aortic dilatation rate, previous aortic dissection, familial history of dissection and sudden death, and the wish to become pregnant, owing to an enhanced risk of rupture during pregnancy [6-11]. Dissection of the aorta, however, also occurs in patients with relatively small aorta diameters, i.e. $<5 \mathrm{~cm}$, without any of the aforementioned risk factors [12]. Therefore, novel prognostic markers are essential to reduce this risk.

Elastic lamina degradation is a hallmark of the diseased MFS aorta, and can be visualized in tissue sections. Notably, elastin fragmentation leads to the release of so-called elastin-derived peptides and fibrillin-1 fragments, containing the hydrophobic GxxPG amino acid sequence, which can bind to the elastin-binding protein (EBP) of the elastin receptor complex (ERC) [13,14]. Signalling via this receptor leads to cellular processes such as proliferation and chemotaxis, but also calcification [15-17]. There is a complex interplay between elastic fibre breaks and calcification, with possibly a vicious cycle whereby elastin breaks lead to calcification, which in turn promotes further fragmentation of the elastic fibres [16,17-23].

Vascular calcification is associated with an increased risk of cardiovascular death $[24,25]$ and has been investigated in many diseases, such as chronic kidney disease, abdominal aortic aneurysms (AAAs), and atherosclerosis [26-29]. In atherosclerosis research, vascular calcification is often categorized into macrocalcification and microcalcification, depending on the size of the calcified lesions [30]. Whereas macrocalcification in arterial tissues is associated with a more stable atherosclerotic plaque phenotype [31], microcalcification is associated with disease progression and plaque rupture [32-36]. Macrocalcification can be easily observed in vascular sections after haematoxylin and eosin (H\&E) staining, whereas the identification of microcalcification requires specific staining methods.

There is a clear lack of knowledge on the possible role of macrocalcification or microcalcification in hereditary aortopathies such as MFS, and their contribution to aortic distensibility and aortic dilatation. In chronic kidney disease and AAA formation, elastin fragmentation promotes vascular calcification $[16,17]$. Recently, elastin-derived peptides were shown to promote AAA formation [37]. Vascular calcification has also been visualized in thoracic aortic aneurysms, specifically in the fibulin-4 hypomorphic and mgR MFS mouse models $[38,39]$. In mgR MFS mice, elastin peptides generated by elastin breaks were shown to contribute to aortic macrophage infiltration, matrix metalloproteinase (MMP) expression, and upregulation of transforming growth factor (TGF)- $\beta$ signalling [40]; however, their relationship with aortic calcification was not investigated. We hypothesize that elastin fragments play a causal role in aortic calcification in MFS, and that microcalcification serves as a novel marker for aortic disease severity.

\section{Materials and methods}

\section{Histological staining}

For histochemical staining, tissue from MFS patients was collected during prophylactic aortic surgery in our institute, to replace the aortic root and ascending aorta. The aortic root and ascending aorta of 2-month-old, 4-month-old and 8-month old Fbn $1^{\mathrm{C} 1039 \mathrm{G} /+} \mathrm{MFS}$ and wild-type (WT) mice were used for histochemical staining. The tissues were fixed with $4 \%$ neutral buffered formaldehyde (Shandon Formal-Fixx; Thermo Scientific, Rockford, IL, USA), paraffin-embedded, sectioned $(7 \mu \mathrm{m})$, deparaffinized in xylene, and rehydrated. Consecutive sections were stained with Alizarin red (Sigma, St. Louis, MO, USA) for calcium deposits, Lawson (Klinipath, Breda, Netherlands) to visualize elastic fibres, Mayer's haematoxylin (Sigma) and eosin (Merck Chemicals, Amsterdam, Netherlands) to stain cellular nuclei/ cytoplasm and macrocalcification, and Alcian blue (Sigma-Aldrich, St. Louis, MO, USA) for detection of glucosaminoglycans (GAGs). The aortic root diameter of the mice was measured as described previously [41]. In brief, the first section of the aortic root, where the valve leaflets were no longer present, was used to measure the aortic root diameter. The luminal diameter was calculated from images of the luminal circumference (using Adobe Photoshop CS5). Macrocalcification in the aortic valve area was measured at $\times 100$ magnification.

Cryosections from the aortic root and the ascending aorta of OsteoSense-800-injected mice were stained with H\&E (macrocalcification) or Lawson (elastic laminae/breaks). The number of elastin breaks was counted by a researcher blinded to the genotype (two sections per mouse). For detection of phosphorylated extracellular signal-regulated kinase-1/2 (pERK1/2), immunohistochemistry was performed on ascending aorta cryosections. First, antigen retrieval was performed (sodium citrate buffer, pH6), followed by endogenous peroxidase quenching $\left(2 \% \mathrm{H}_{2} \mathrm{O}_{2}\right)$, and non-specific antigen blocking with superblock (ScyTek) was performed prior to overnight incubation with rabbit polyclonal anti-pERK1/2 antibody (1:200, \#9101; Cell Signaling Technology, Leiden, Netherlands). The sections were then incubated with a horseradish peroxidaseconjugated polymer anti-rabbit IgG, and subsequently developed with 3,3'-diaminobenzidine tetrahydrochloride and photographed/quantified with QWin software (Leica Microsystems, Cambridge, UK) as the positive area/total medial area ratio.

\section{Smooth muscle cell (SMC) culture}

Primary human umbilical artery SMCs were isolated [42] and cultured in Dulbecco's modified Eagle's medium F-12 with L-glutamine, $100 \mathrm{U} / \mathrm{ml}$ penicillin, $100 \mu \mathrm{g} / \mathrm{ml}$ streptomycin, and $10 \%$ fetal bovine serum (FBS). Murine aortic SMCs were isolated from 5-week-old Fbn1 ${ }^{\mathrm{C} 1039 \mathrm{G} /+}$ and WT aortas [43], and cultured with $20 \%$ FBS. Cell experiments were initiated 
at $80 \%$ confluency. SMCs were incubated for 6 or 12 days with $100 \mu \mathrm{g} / \mathrm{ml}$ elastin peptide VGVAPG, scrambled peptide VVGPGA (Genecust, Ellange, Luxembourg), or vehicle. WT aortic SMCs were also treated with a combination of elastin peptides and $5 \mathrm{~mm}$ lactose, which causes shedding of EBP to block ERC signalling [13], or with elastin peptides and $25 \mu \mathrm{M}$ PD98059 [mitogen-activated protein kinase kinase 1/2 (MEK1/2) inhibitor (MEKi); Sigma] to inhibit MEK1/2. Additionally, cells were treated with $300 \mu \mathrm{g} / \mathrm{ml} \kappa$-elastin (Elastin Products Company, Owensville, Missouri USA), i.e. elastin fragments purified from bovine ligament that contain the VGVAPG sequence.

Simultaneous treatment with $\kappa$-elastin and lactose or with $\kappa$-elastin and $400 \mu \mathrm{M}$ neuraminidase inhibitor 2,3-dehydro-2-deoxy- $N$-acetylneuraminic acid (ddNeu5ac) was also performed to block ERC signalling. Finally, WT and MFS SMCs were separately treated with $5 \mathrm{mM}$ lactose or 40-80 $\mu \mathrm{M}$ PD98059.

\section{RNA isolation and reverse transcription quantitative polymerase chain reaction (RT-qPCR)}

RNA was isolated (Aurum Total RNA Mini Kit; BioRad, Veenendaal, Netherlands), and cDNA synthesis was performed (iScript cDNA synthesis kit; BioRad). Specific primers were designed (software: Primer3) to analyse the gene expression of different calcification markers (supplementary material, Table S1). The 60S acidic ribosomal protein $\mathrm{P} 0$ gene ( $R P L P O)$ was used as the reference gene (supplementary material, Table S1). Quantitative polymerase chain reaction was performed on a Lightcycler 480 system (Sensifast no ROX SYBR green mix; Bioline, London, UK).

\section{Protein isolation and western blot analysis}

Murine aortic tissue was lysed with RIPA buffer containing $150 \mathrm{mM} \mathrm{NaCl}, 50 \mathrm{mM}$ Tris- $\mathrm{HCl}, 1 \%$ Igepal-39, $0.5 \%$ sodium deoxycholate, $0.1 \%$ sodium dodecyl sulphate (SDS), and phosphatase and protease inhibitor. The supernatant was collected after $10 \mathrm{~min}$ of centrifugation $(8000 \times g)$. The protein concentration was measured (BioRad DC assay), an equal amount of protein was loaded onto a $10 \%$ SDS polyacrylamide gel electrophoresis gel, and protein transfer was performed on a polyvinylidene difluoride membrane. pERK1/2 was detected with a mouse monoclonal IgG2a (sc-7383; Santa Cruz, Dallas, TX, USA), and total extracellular signal-regulated kinase-1/2 (ERK1/2) was detected with a rabbit polyclonal IgG (\#9102; Cell Signaling Technology). $\alpha$-Tubulin was used as the loading control (CLX135AP; Cedarlane, Burlington, Canada). All antibodies were diluted 1:1000. Finally, the blots were incubated with the corresponding secondary IRdye-labelled antibody (LI-COR, Lincoln, NB, USA) and visualized with the Odyssey Infrared Imaging System (LI-COR).

\section{Alkaline phosphatase (ALP) activity}

For detection of in vitro ALP activity, cells were lysed with cold RIPA buffer containing protease inhibitors.
Lysates were incubated overnight at $4{ }^{\circ} \mathrm{C}$, and diluted $1: 1$ with Vector blue ALP substrate (Vector, Peterborough, $\mathrm{UK})$ in $400 \mathrm{~mm}$ Tris-HCl (pH 8). Finally, the optical density was measured with an EL-808 ultra-microplate reader at a wavelength of $650 \mathrm{~nm}$. ALP activity was corrected for the protein concentration of the lysate.

\section{Animal experiments}

Animal care and experimental procedures were approved by the independent animal experimental committee for Animal Welfare of the Academic Medical Centre and the Erasmus Medical Centre according to the local guidelines and Directive 2010/63/EU of the European Parliament. To study vascular macrocalcification, 2-month-old, 4-month-old and 8-month-old Fbn $1^{\mathrm{C} 1039 \mathrm{G} /+}$ mice (MFS; $n=114$ ) and WT mice $(n=38)$ were analysed. The mice are maintained on a C57B16/J background as a heterozygous breeding colony in our mouse facility. Eight-month-old Fbn $1^{\text {C1039G/+ }}$ male and female mice $(n=11)$ were used for the in vivo near-infrared fluorescence (NIRF) study with OsteoSense-800 and MMPSense-680 (PerkinElmer, Boston, MA, USA). Eight-month-old WT littermates $(n=7)$ were included as a reference group. The mice were injected twice (1 and 3 days prior to imaging; intraperitoneal injections) with the fluorescent bisphosphonate OsteoSense-800 probe, and once (1 day prior to imaging; intravenous injection) with the MMPSense-680 probe ( $2 \mathrm{nmol} / 25 \mathrm{~g}$ body weight). In vivo imaging was performed under isoflurane anaesthesia (4\% isoflurane for induction; $3 \%$ isoflurane for maintenance). NIRF imaging was performed with a fluorescence tomography device (FMT, 2500XL; PerkinElmer). Transthoracic echocardiography was performed on a subset of mice at the Erasmus Medical Centre with a Vevo2100 high-resolution imaging system and a 32-56-MHz probe to measure the aortic (root and ascending) diameter and distensibility (RMV704 scanhead; VisualSonics, Toronto, Canada). The remaining mice underwent ultrasound investigation at the Academic Medical Centre, with a Vevo770 high-resolution imaging system and a 40-MHz probe (RMV704 scanhead; VisualSonics). The distensibility was calculated by subtracting the measured ascending aortic diameter in diastole from the systolic aortic diameter, and then dividing it by the diastolic aortic diameter (WT, $n=4$; MFS, $n=9$ ). After the mice had been killed, the heart and aorta were perfused with saline, fixed, and imaged on the Odyssey Infrared Imaging System. Intensity was assessed with ImageJ.

\section{Statistical analysis}

Statistical tests were performed with GraphPad Prism 5 software. Graphs represent mean \pm standard error of the mean. To analyse differences between two groups, Student's $t$-test or the Mann-Whitney $U$-test was used, as appropriate. Proportions were analysed with Fisher's exact test. Association analysis was performed with 

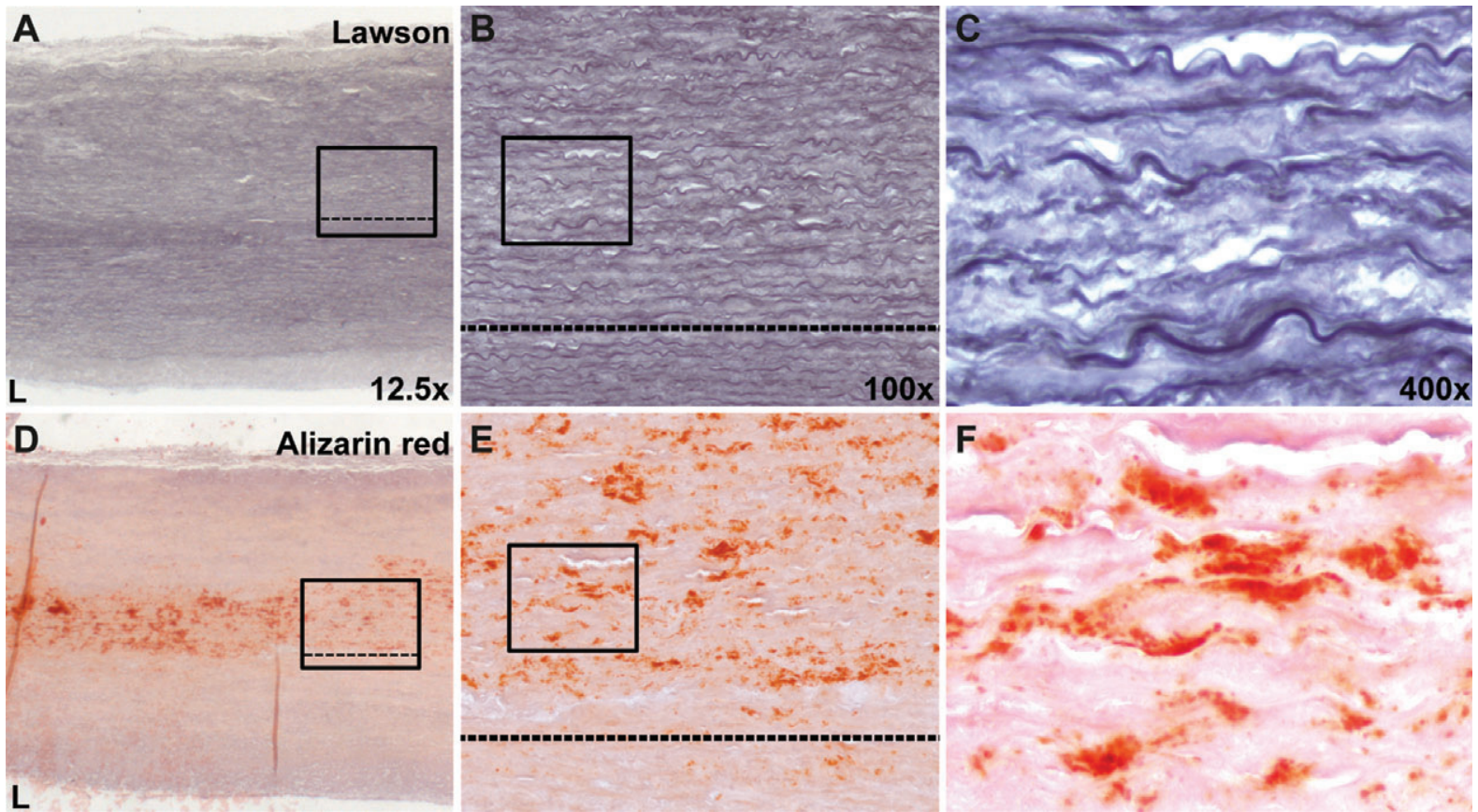

Figure 1. Elastin breaks and microcalcification in the ascending aorta of an MFS patient. (A) Elastin in the dilated ascending aorta of an MFS patient was visualized by Lawson staining of tissue sections. Elastin fragmentation was most pronounced in the middle of the media, resulting in less intense staining (magnification: $\times 12.5$ ). (B) The boxed area in (A) is shown at greater magnification with extensive elastin breaks above the dotted line (magnification: $\times 100$ ) [detail from (A)]. (C) Photomicrograph depicting a detail of elastic lamina damage (magnification: $\times 400$ ) [boxed area in (B)]. (D) In a consecutive section, microcalcification was visualized by Alizarin red staining. The area of microcalcification co-localized in the middle of the aortic media with the area of most extensive aortic wall damage, as shown in (A) (magnification: $\times 12.5)$. (E) Calcium deposits were localized in the same area as in (B), where extensive elastin breaks are present (magnification: $\times 100$ ) [detail from (D)]. (F) Photomicrograph depicting a detail of the calcium deposits (magnification: $\times 400)$ [boxed area in (E)].

Pearson's correlation test. A $P$ value of $\leq 0.05$ was considered to be statistically significant.

\section{Results}

\section{Microcalcification co-localizes with elastic lamina fragmentation in MFS}

To study the co-localization between the loss of elastin fibre integrity and vascular calcification in the human MFS aorta, sections of the ascending aorta of an MFS patient were stained for elastin (Lawson) and calcium deposits (Alizarin red). In this aortic section, areas of extensive elastin fragmentation showed reduced Lawson staining primarily in the centre of the aortic media (Figure 1A; larger magnifications in Figure 1B, C). We observed microcalcification in the ascending aorta as visualized by Alizarin red staining (Figure 1D-F). The microcalcification in the centre of the aortic media colocalized with the area of reduced elastic fibre integrity.

\section{Elastin peptides induce SMC calcification}

Elastin fragmentation, as observed in the MFS aorta, leads to the formation of elastin peptides [44] that can interact with ERC [13]. ERC consists of EBP, which is a splice variant encoded by the $\beta$-galactosidase- 1 gene (GLB1) [15], cathepsin A (CTSA), and neuraminidase-1 (NEU1). ERC mediates signalling, resulting in cellular processes such as proliferation and differentiation, as well as increased cytokine and protease expression [15], and calcification $[21,23]$. We found that $G L B 1$, which encodes $\beta$-galactosidase- 1 and EBP, is expressed in human arterial SMCs (supplementary material, Figure S1A), and that the specific mRNA transcript variant encoding EBP (GLBl variant 2) is also present (supplementary material, Figure S1B). In addition, CTSA and NEU1 are expressed (supplementary material, Figure S1C, D). To study the effect of elastin peptides on vascular calcification in detail, SMCs were treated with the elastin peptide VGVAPG containing the GxxPG consensus sequence, which is repeated frequently in tropoelastin [45], or with the peptide VVGPGA as a scrambled control.

In SMCs, elastin peptide treatment caused a 1.8-fold increase in tissue-non-specific ALP (ALPL) mRNA (Figure 2A). ALP is a protein that promotes vascular calcification by decreasing the level of the mineralization inhibitor pyrophosphate (PPi) [46,47]. There was also a two-fold decrease in the mRNA level of matrix GLA protein (MGP) (Figure 2B), which inhibits calcification by antagonizing bone morphogenetic protein (BMP) and by binding to hydroxyapatite $[48,49]$. These results indicate an induced calcification process.

\section{ALP activity is induced via ERK $1 / 2$}

In murine aortic SMCs, the elastin peptides induce a two-fold increase in ALP activity (Figure 2C). To assess 
A
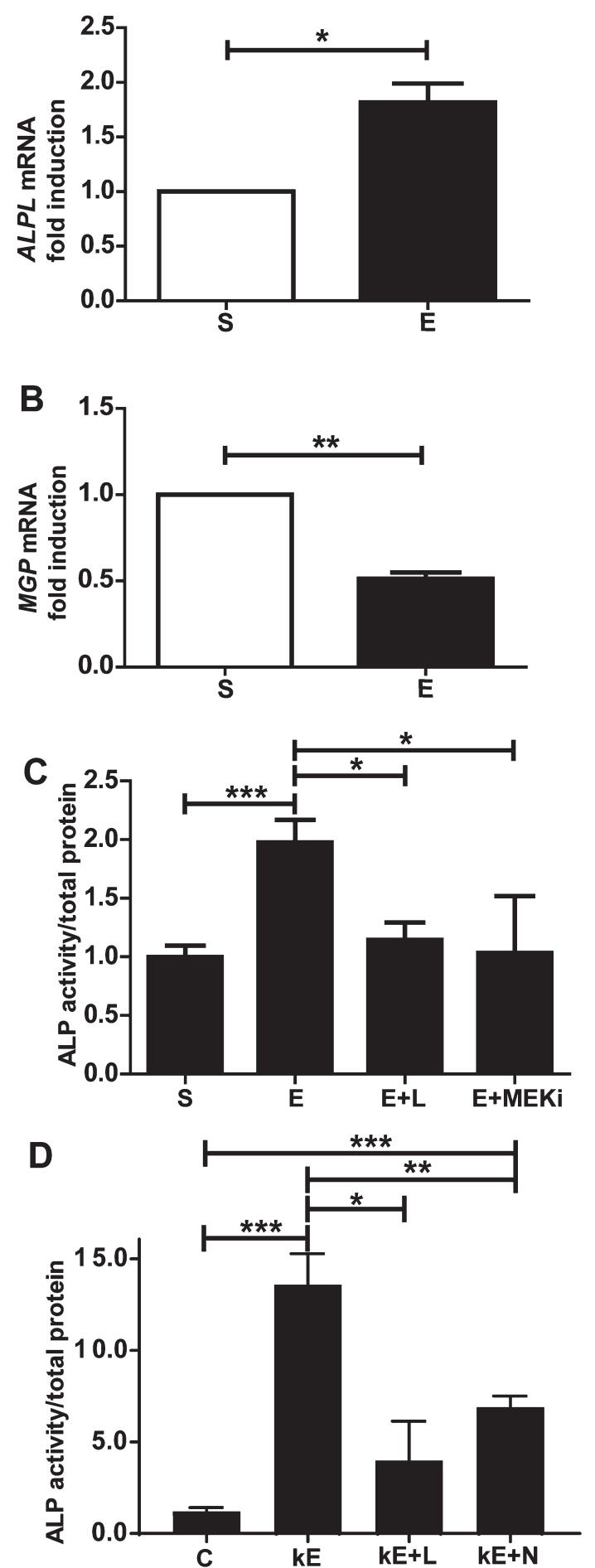

E
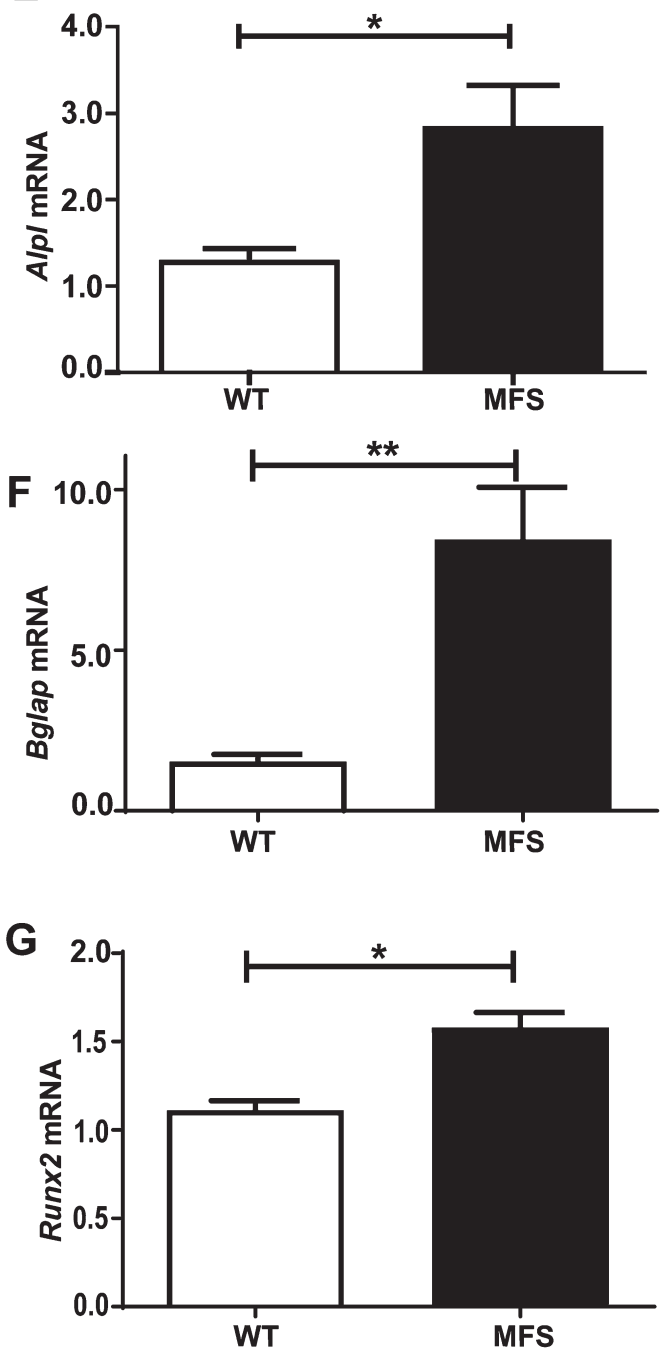

H

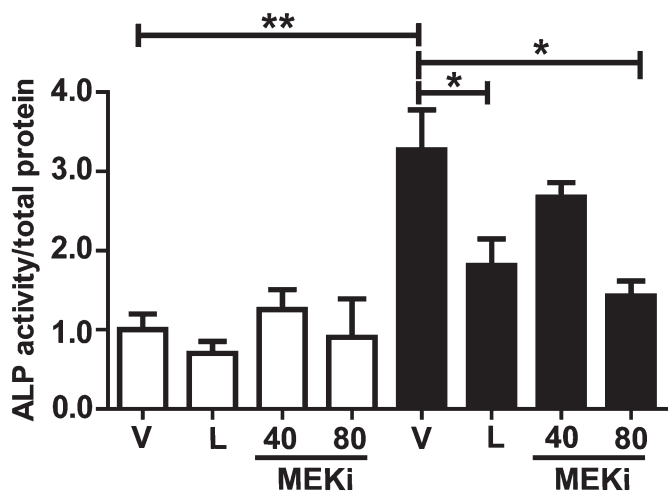

Figure 2. Elastin peptides enhance ALP activity in MFS SMCs via ERC and ERK1/2 signalling. (A) Elastin-derived peptides upregulated ALP liver/bone/kidney (ALPL) mRNA in human SMCs ( $n=9$ wells per group). (B) Matrix GLA protein (MGP) mRNA expression was downregulated in human SMCs by the elastin peptides. (C) In WT aortic SMCs, elastin-derived peptide (E) treatment increased ALP activity (normalized for protein content) as compared with scrambled peptides (S) (optical density units per $\mathrm{mg} / \mathrm{ml} ; n=12$ ). Simultaneous treatment with lactose (L), an ERC antagonist, or MEKi, an inhibitor of the ERK1/2 pathway, attenuated the effect of elastin peptides on ALP activity $(n=6-9)$. (D) Similarly, $\kappa$-elastin ( $\mathrm{kE}$ ) treatment upregulated ALP activity as compared with control (C), and this effect could be partially blocked by lactose (L) or ddNeu5ac $(N)$, a neuraminidase inhibitor $(n=3)$. (E) Alp/ mRNA Arbitrary Unit (AU) expression was increased in MFS aortic SMCs $(n=6)$ as compared with WT SMCs $(n=4)$. (F and G) Bone $\gamma$-carboxyglutamic acid-containing protein $($ Bglap) mRNA (F) and runt-related transcription factor-2 (Runx2) mRNA (G) were also upregulated 5.5-fold and 1.4-fold, respectively, in MFS aortic SMCs. (H) The basal ALP activity was increased in murine MFS (black bars) versus WT (white bars) aortic SMCs $(n=15)$. Treatment of MFS murine SMCs with lactose $(\mathrm{L})$ or MEKi reduced basal ALP activity dose-dependently $(n=6-9)$ as compared with vehicle-treated cells $(\mathrm{V}) .{ }^{*} P<0.05$, ${ }^{* *} P \leq 0.01,{ }^{* *} P<0.001$. 
whether the enhanced ALP activity was mediated via activation of ERC, we applied lactose, which is a known antagonist of ERC by shedding of EBP [13]. The elastin peptide-induced ALP activity was indeed attenuated by lactose treatment (Figure 2C). Similarly, this was observed when more natural elastin fragments were used, so-called $\kappa$-elastin, purified from bovine ligament (Figure 2D). In addition, more specific inhibition of ERC was shown when ddNEU5ac was used to block NEU1; this suppressed the upregulation of ALP activity, accentuating the involvement of ERC in the calcification process (Figure 2D). To further investigate which signalling pathway is involved, SMCs were treated with a MEK1/2 inhibitor (MEKi), to prevent ERK1/2 phosphorylation. MEKi blocked the increase in ALP activity (Figure 2C), indicating that the ERK1/2 pathway is involved in elastin peptide-mediated signalling.

\section{ERC and ERK $1 / 2$ signalling contribute to MFS-related SMC calcification}

The role of ALP in MFS-related calcification was investigated by comparing WT and MFS aortic SMCs. The Glb1, Ctsa and Neul genes, encoding components of ERC, are expressed in murine aortic WT and MFS SMCs, and Glb1 expression and Ctsa expression are enhanced in MFS SMCs (supplementary material, Figure S1E-G). Interestingly, MFS SMCs showed an enhanced osteogenic profile by an increase in basal mRNA levels of the genes Alpl, bone $\gamma$-carboxyglutamic acid-containing protein (Bglap), and runt-related transcription factor-2 (Runx2) (increased two-fold, 5.5-fold and 1.4-fold, respectively) (Figure $2 \mathrm{E}-\mathrm{G}$ ). In line with these data, ALP activity was increased three-fold in MFS SMCs (Figure 2H), whereas these cells were not stimulated by elastin peptides. Lactose treatment reduced ALP activity in the MFS SMCs (Figure 2H), suggesting enhanced elastin-mediated signalling in MFS SMCs in culture. Blockade of ERK1/2 signalling by MEKi also reduced ALP activity in a dose-dependent fashion in MFS SMCs (Figure 2H).

Here, we show that elastin peptides promote an SMC calcification phenotype, and that SMCs derived from MFS mice show increased basal ALP activity, which can be blocked by inhibition of ERC and ERK1/2 signalling. These data are in line with the observation in human MFS aorta that microcalcification co-localizes with elastin degradation.

\section{Macrocalcification is prominent in the murine MFS aortic root}

So far, calcification studies in MFS mice have been limited. Upon close inspection of aortic root histology (H\&E staining), overt signs of macrocalcification were frequently observed in MFS mice (Figure 3). GAGs are long unbranched polysaccharides that are known to be abundant in cartilage and bone, and to accumulate in areas of vascular calcification [38,39,50,51]. Detection of GAGs by Alcian blue staining revealed that, predominantly, the SMC-containing medial area becomes calcified in MFS mice (Figure 3A). To visualize calcium deposits, we applied Alizarin red, which revealed acellular calcium deposits and deposition surrounding osteoblast-like cells (Figure 3A).

To evaluate whether macrocalcification in the aortic root is different between WT and MFS mice, we quantified the percentage of mice with osteoblast-like cells per age group. Vascular calcification in the aortic root increased with age only in MFS mice, with prevalence rates of $10 \%$ in 2-month-old mice and $70 \%$ in 8-month-old mice (Figure 3B). This did not occur in WT mice, with prevalence rates of $10 \%$ in in 2-month-old mice and $11.1 \%$ and 8 -month-old mice (Figure 3B). There was a modest significant correlation between the area of calcium deposits and/or osteoblast-like cells, and aortic root diameter in 4-month-old mice (Figure 3C). In conclusion, macrocalcification in the aortic root of MFS mice is strongly enhanced, but there is only a modest correlation with aortic dilatation.

\section{Microcalcification is abundant in the ascending aorta of MFS mice}

To study calcification in vivo, we used the NIRF bisphosphonate probe OsteoSense-800, which is known to bind to hydroxyapatite. This probe has already successfully been used in mice with atherosclerosis to reveal calcification in atherosclerotic plaques [52]. The OsteoSense-800 probe was injected into 8-month-old WT and MFS mice, for subsequent monitoring of active incorporation in the vasculature. A positive signal was observed in the heart area in vivo, but it was not detailed enough for quantification (supplementary material, Figure S2A). On closer inspection, OsteoSense-800 signal could be observed in the aortic root, and did indeed co-localize with macrocalcification (supplementary material, Figure S2B). Upon ex vivo imaging of the heart and entire aorta, the OsteoSense- 800 probe revealed extensive calcification (green) in the ascending aorta of MFS mice (Figure 4A), whereas not much NIRF signal was observed in the arch and descending aorta (Figure 4B). In comparison, the MMPSense-680 probe, used to visualize MMP activity, was abundant throughout the heart and ascending aorta in both WT and MFS mice, and there was no significant difference between the groups (supplementary material, Figure S2C, D). To further investigate the calcified ascending aorta, sections were studied. The OsteoSense- 800 probe was visible in the medial area at sites with extensive elastin breaks (Figure 4C; elastin appears as red autofluorescence), which is similar to what was observed in MFS patients (Figure 1). Interestingly, these calcium deposits could only be visualized by the OsteoSense- 800 probe. As there were no overt signs of large acellular calcium deposits or osteoblast-like cells, the vascular calcification process in the ascending aorta may be considered to be microcalcification [53]. To assess whether aortic microcalcification is enhanced in MFS mice, we quantified the intensity of OsteoSense-800 in whole-mount ascending aorta, and this revealed a significant increase 
A

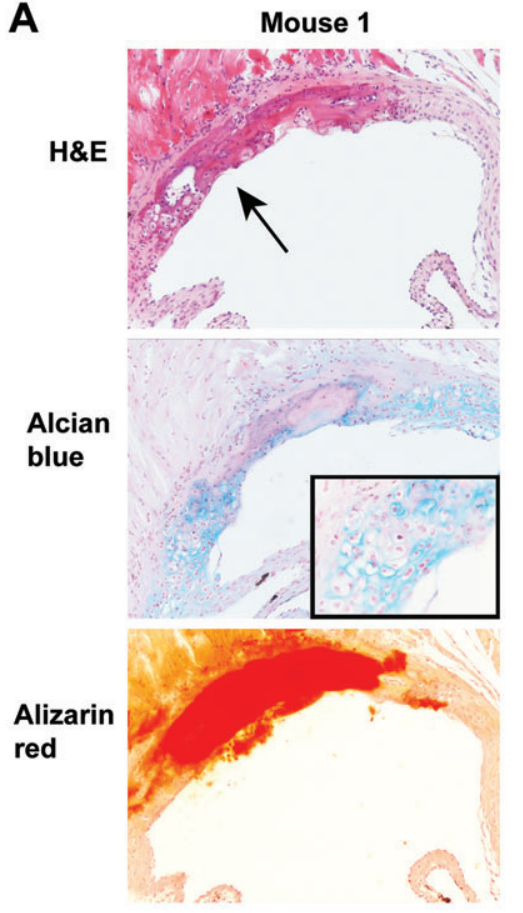

B

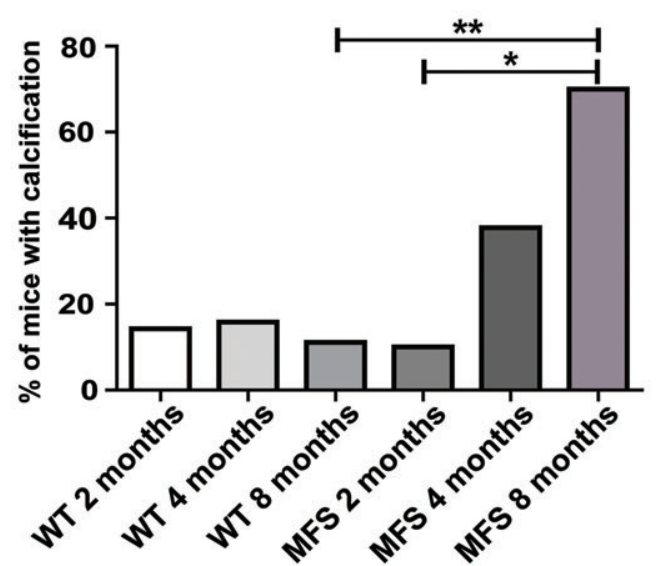

Mouse 2

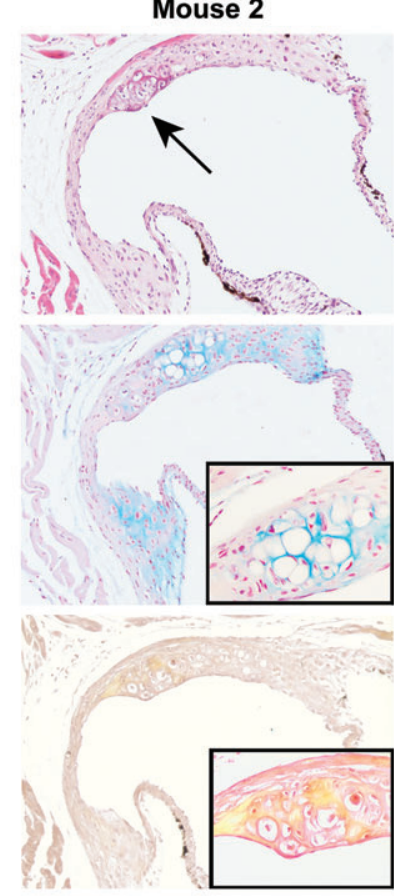

Mouse 3

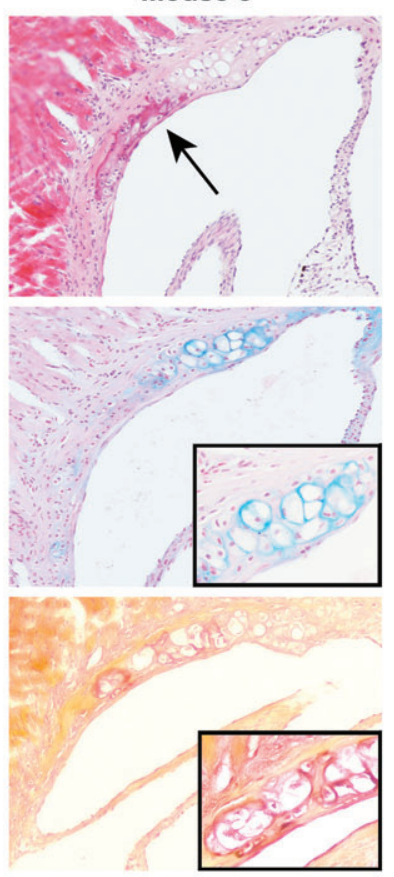

C

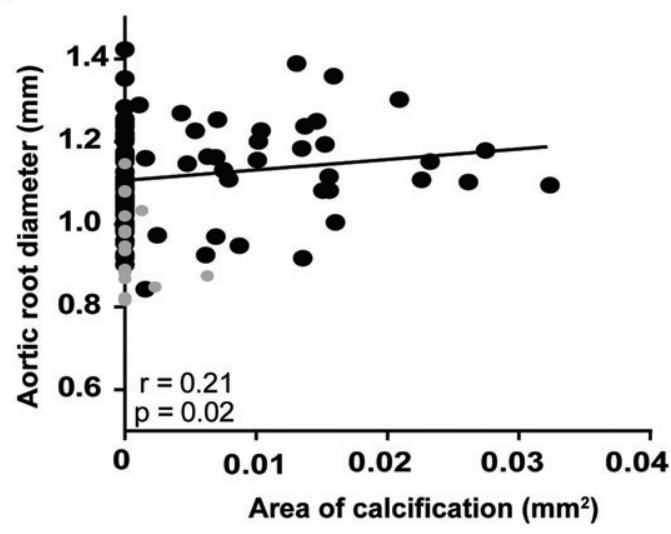

Figure 3. Macrocalcification in the murine MFS aortic root. (A) Photomicrographs of aortic root sections of three MFS mice are depicted (magnification: $\times 100$ ). Consecutive sections were stained with H\&E, Alcian blue, and Alizarin red. Osteoblast-like cells were observed in the aortic medial layer (arrows, upper panels) of the MFS mice. GAGs (Alcian blue; middle panels) and calcium deposits (Alizarin red; lower panels) surrounded these bone-like cells. Insets show osteoblast-like cells in more detail (magnification: $\times 400)$. (B) In MFS mice ( $n=114)$, the percentage of mice with calcification increased with age, whereas this was not observed in WT mice $(n=38)$. (C) There was a weak but significant correlation between the area of calcification in the aortic root and aortic root diameter (grey dots are WT mice and black dots are MFS mice). ${ }^{*} P<0.05,{ }^{* *} P \leq 0.01$.

in microcalcification in MFS mice as compared with WT mice (Figure 4D). In conclusion, microcalcification is more abundant in the MFS ascending aorta in close proximity to elastin fragmentation.

\section{ERK $1 / 2$ signalling is correlated with aortic} microcalcification

As mentioned above, ERK1/2 inhibition resulted in a decrease in ALP activity after elastin peptide stimulation and in murine MFS SMCs. Here, we show that $\mathrm{pERK} 1 / 2$ was significantly increased in the MFS ascending aorta as compared with WT ascending aorta lysates (Figure 5A, B). To determine where the enhanced ERK1/2 signalling is present in the aorta and whether there is a relationship with aortic microcalcification, immunostaining for pERK1/2 was performed on aorta sections from the OsteoSense-800-injected mice. Enhanced pERK1/2 was observed in SMCs in the aortic media, and correlated significantly with the OsteoSense-800 signal (Figure 5C, D; $r=0.62$, $P=0.02)$.

Aortic dilatation, distensibility and elastin breaks are correlated with aortic microcalcification

As previous studies have shown that ERK1/2 signalling plays a pivotal role in the progression of MFS aortic 
A

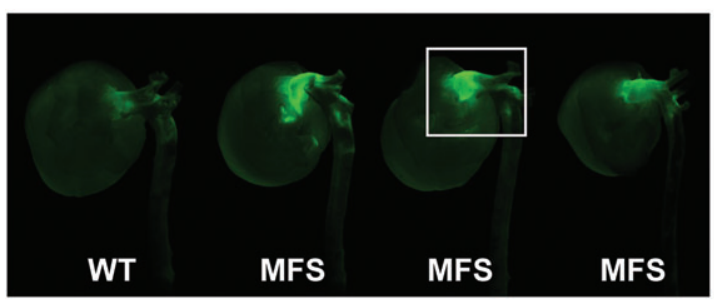

C

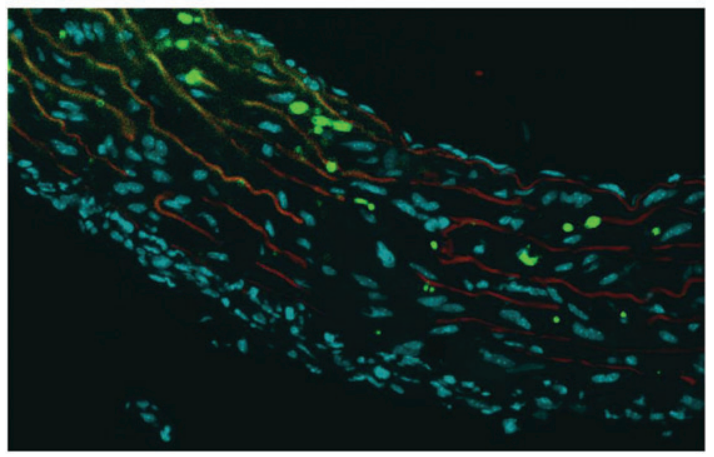

B

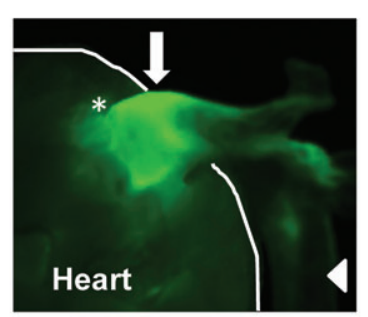

D

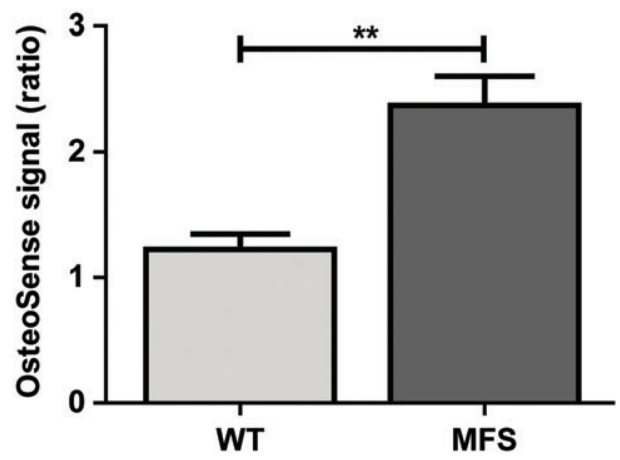

Figure 4. Microcalcification in the MFS mouse ascending aorta. (A) OsteoSense-800, a bisphosphonate-conjugated fluorescent probe, was injected intraperitoneally into WT and MFS mice. This probe binds to sites of vascular calcification (green). The aortas of WT and MFS mice were harvested, and the OsteoSense-800 was visualized ex vivo with an Odyssey scanner. (B) Examples of one WT mouse and three different MFS mice. The boxed area in (A) (white frame) is shown at greater magnification. The OsteoSense-800 (green) localized in the ascending aorta (arrow), originating from the aortic root (*). There was minimal signal in the descending aorta (arrowhead). (C) A section of the ascending aorta of an MFS mouse revealed accumulation of the OsteoSense-800 (green) in the medial layer of the vascular wall. Cell nuclei were stained blue, and the elastic laminae showed red autofluorescence. (D) Significantly more fluorescence was incorporated in the MFS $(n=11)$ than in the WT $(n=7)$ ascending aortas, as quantified from the whole mount scans (as fold induction relative to WT mice). ${ }^{* *} P \leq 0.01$.

disease [54-56], it is important to describe the various pathological features in relation to microcalcification. Aortic dilatation of the ascending aorta, as determined by echocardiography, correlated positively with microcalcification (Figure 5E; $r=0.55, P=0.050$ ). In MFS, a known risk factor for aortic growth/dissection is aortic distensibility [57], which is a marker for aortic elasticity. When the distensibility decreases, it is considered that the elastic laminae are compromised and that the aorta has therefore become more stiff [57]. Indeed, upon quantification, the number of elastin breaks was six-fold higher in the MFS mouse aorta where the distensibility was the lowest (supplementary material, Figure S3). Interestingly, in MFS mice, a strong negative correlation between calcification and distensibility was observed (Figure 5F; $r=-0.71, P=0.006$ ). In addition, a positive correlation between calcification and the number of elastin breaks was observed (Figure $5 \mathrm{G}, \mathrm{H} ; r=0.65, P=0.021$ ), which is in line with our in vitro experiments showing enhanced ALP activity after elastin peptide treatment. On the basis of these data, microcalcification may be considered a marker for local aortic damage in MFS mice. In summary, we propose that elastin fragmentation in the MFS aorta leads to increased microcalcification via activation of ERC and subsequently ERK1/2 signalling, resulting in enhanced ALP activity and microcalcification (Figure 6).

\section{Discussion}

In the current study, we observed extensive microcalcification, not macrocalcification, in the human MFS ascending aorta. In line with our data, aortic calcification in MFS patients is not visible on a computed tomography (CT) scan. On histochemical staining in the MFS aorta, microcalcification became apparent and co-localized with areas of severe elastin fragmentation, suggesting a causative relationship. Regarding this hypothesis, we demonstrated that elastin peptides induced a calcification process in cultured human and mouse arterial SMCs as revealed by enhanced ALP and reduced MGP expression. In addition, cultured murine MFS aortic SMCs showed enhanced Alpl, Bglap and Runx 2 mRNA expression and increased basal ALP activity, confirming the osteogenic potential of these SMCs. Study of macrocalcification and microcalcification in ageing MFS mice revealed that macrocalcification in the aortic root correlated only mildly with aortic diameter, whereas microcalcification in the ascending aorta was strongly associated with aortic diameter, distensibility, and elastin degradation.

Vascular calcification is controlled by balancing extracellular calcium and phosphate levels versus the PPi level, which are regulated by multiple proteins; ALP is known to decrease the PPi level [46], and MGP reduces the level of the osteogenic growth factor BMP 
A

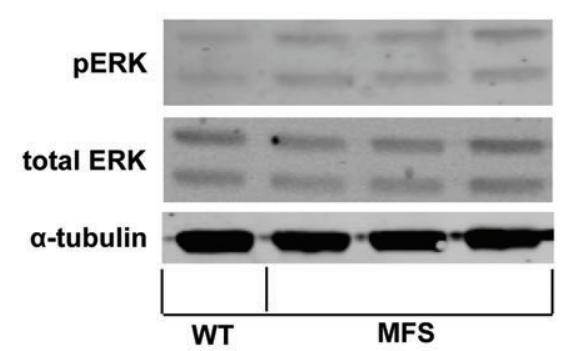

C

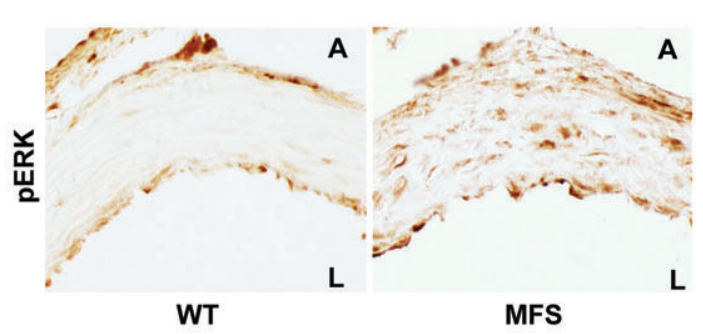

E

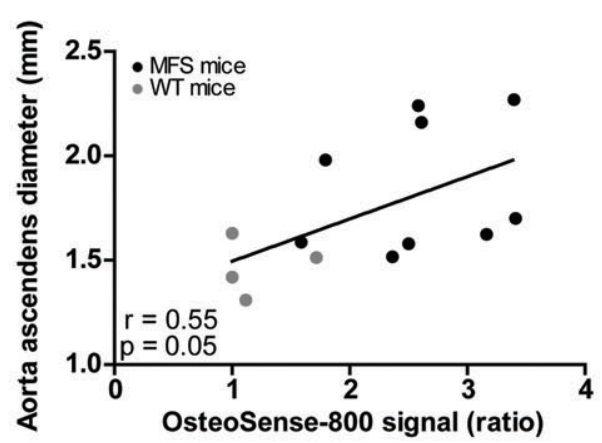

G

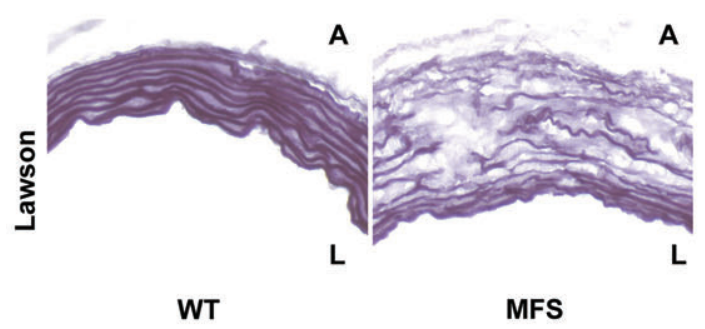

B
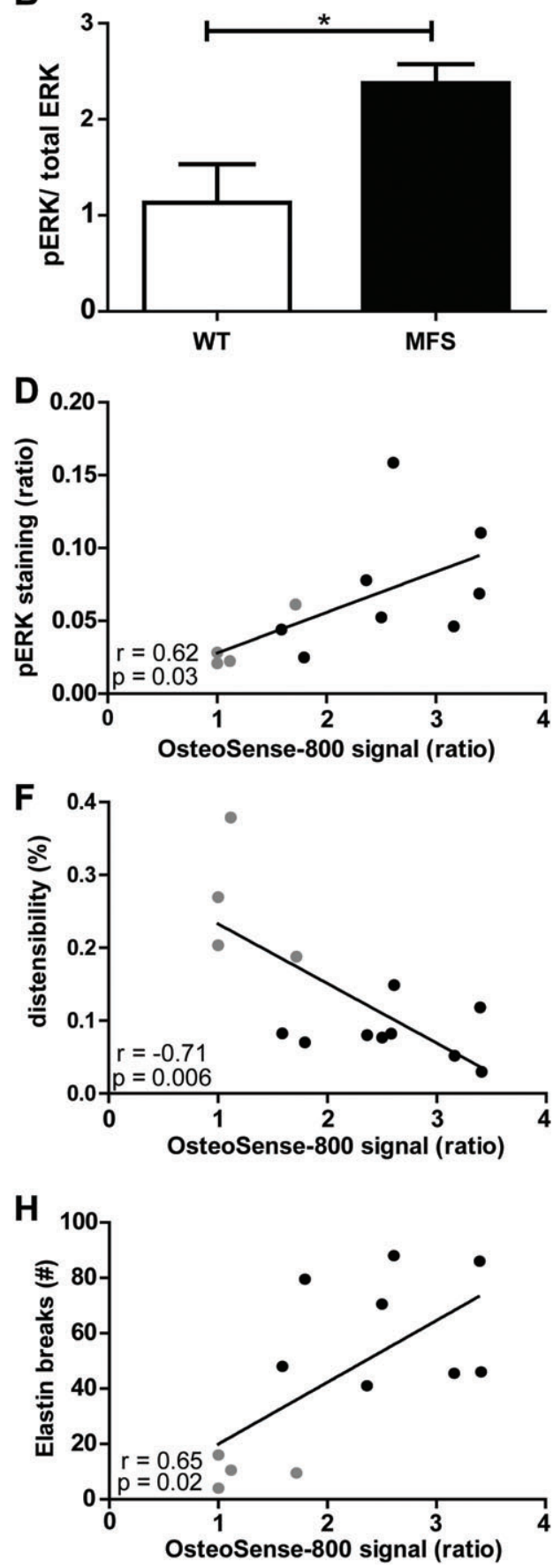

Figure 5. Aortic diameter, distensibility, elastin breaks and pERK1/2 in relation to microcalcification. (A) Western blot for $p$ ERK1/2, total ERK1/2 and $\alpha$-tubulin in WT and MFS ascending aortas. (B) The pERK1/2 level (corrected for total ERK1/2) was significantly higher in the MFS ascending aorta $(n=7)$ than in the WT ascending aorta $(n=5)$. (C) Representative photomicrographs of pERK $1 / 2$ immunostaining of the ascending aorta of a WT mouse and an MFS mouse (magnification: $\times 200$ ). A, adventitia; L, lumen. (D) pERK1/2 staining correlated positively with the OsteoSense-800 signal. (E) The amount of OsteoSense-800 accumulation correlated with ascending aorta diameter (in $\mathrm{mm}$ ) (grey dots are WT mice and black dots are MFS mice). (F) There was a strong negative correlation between the OsteoSense- 800 signal and ascending aorta elasticity, also known as distensibility (\%). (G) Representative photomicrographs of the elastic laminae as visualized by Lawson staining of the ascending aorta of a WT mouse and an MFS mouse (magnification: $\times 200)$. $A$, adventitia; $L$, lumen. $(H)$ The number of elastin breaks in the aorta correlated positively with the OsteoSense-800 signal. ${ }^{*} P<0.05$.

and also directly binds to hydroxyapatite $[48,49]$. In our SMC cultures, we observed a disturbed balance, with enhanced ALP and reduced MGP expression in response to elastin peptide stimulation. MGP-deficient mice have extensive spontaneous arterial calcification [58], which can be reduced by BMP inhibitors [59].
Elastin haploinsufficiency can also inhibit arterial calcification in MGP-deficient mice, independently of BMP signalling. The latter is explained by the function of elastin as an accelerator of calcification [19]. In line with our data, it was demonstrated that elastin peptides induced ALP activity in rat SMCs and 


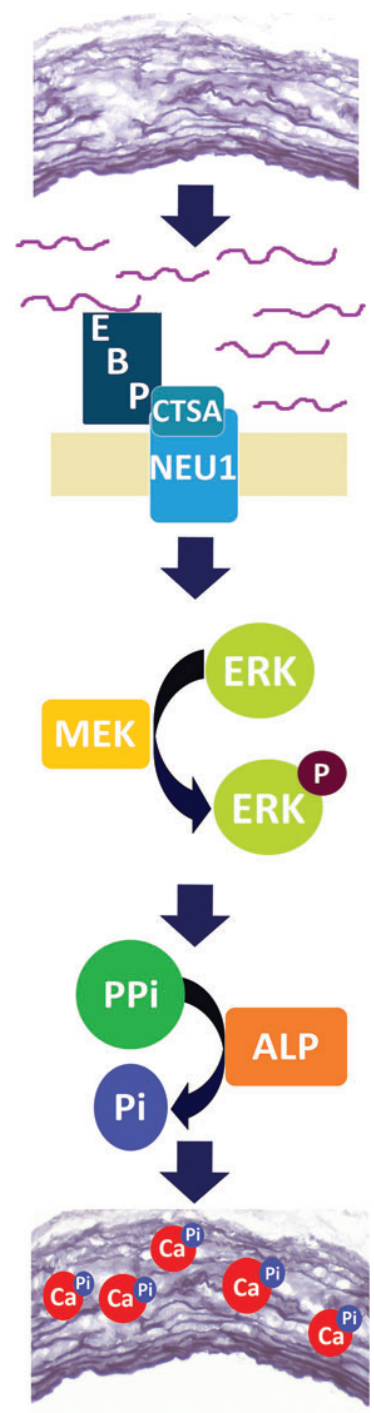

Figure 6. Mechanism underlying MFS aortic microcalcification: schematic overview of the main findings of this study. Elastin peptides (purple strings) generated by local aortic elastin fragmentation bind to ERC, which consists of EBP, CTSA, and NEU1. This causes ERK $1 / 2$ phosphorylation via MEK $1 / 2$, which leads to an ERK1/2-dependent increase in ALP activity. ALP causes hydrolysis of $\mathrm{PPi}$, an inhibitor of calcification, and generates phosphate (Pi). Calcium phosphate crystals, in the form of hydroxyapatite, precipitate, causing microcalcification in the MFS aorta, which functions as a marker for aortic damage and may increase the risk of aortic rupture.

fibroblasts [21,22] and increased ERK1/2 signalling $[13,60]$. Moreover, the ERK1/2 pathway is profoundly involved in aggravating MFS aortic disease [54-56,61]. Other studies have already linked ERK1/2 signalling to vascular calcification $[62,63]$. Here, we showed that elastin peptides increased ALP activity in an ERC-dependent and ERK1/2-dependent manner in SMCs. Basal ALP activity was already enhanced in MFS aortic SMCs, and could be normalized by the ERC and ERK1/2 inhibitor lactose, the neuraminidase inhibitor ddNeu5ac, and MEKi. In the Fbn $1^{\mathrm{C} 1039 \mathrm{G} /+}$ MFS mouse model, we observed macrocalcification with large calcium deposits and osteoblast-like cells in the medial area of the aortic root, which was almost absent in
WT mice. In addition, microcalcification was revealed in the MFS ascending aorta with the NIRF probe OsteoSense-800. Hypomorphic $\mathrm{mgR} / \mathrm{mgR}$ MFS mice are known to have increased medial microcalcification in the ascending aorta [38], and it would be of interest to study whether macrocalcification is present in the aortic root of these mice. In contrast, macrocalcification is present in the aortic root of fibulin- $4^{\mathrm{R} / \mathrm{R}}$ mice [39], but, in this mouse model, microcalcification in the ascending aorta was never studied. Altogether, these data indicate potential common signalling pathways between the distinct genetic aneurysm mouse models in which different extracellular matrix proteins are mutated. It is of note that both fibulin-4 and fibrillin-1 are necessary for proper elastin fibre assembly [64], and lack of either of these proteins may impair elastin homeostasis.

The relationship between elastin fragmentation and calcification has been observed before. Elastin fragmentation precedes vascular calcification [17,18,21-23], but calcium crystals can also cause damage to elastin fibres [19,20]. In addition, cellular vesicles, derived from apoptotic cells, can induce vascular calcification in atherogenic mouse models [65], thereby causing elastin degradation. Moreover, TGF- $\beta$ increases ALP activity and microcalcification via collagen receptor discoidin domain receptor-1-mediated vesicle release by SMCs [66]. As enhanced TGF- $\beta$ signalling is also a hallmark of MFS pathology, together with elastin fragmentation and SMC death, these processes may all be relevant and act synergistically to cause aortic calcification and further aortic damage in MFS.

In atherosclerosis, microcalcification is associated with enhanced atherosclerotic plaque rupture [33-36,67], and thus contributes to enhanced morbidity and mortality. A causal role for microcalcification during aneurysm formation has become clear from the adapted calcium phosphate-induced AAA model, with enhanced aneurysm growth as compared with the original calcium chloride model [68]. Further studies are needed to determine whether inhibition of aortic calcification with, for example, an ALP inhibitor [47] could serve as therapeutic option for MFS aortic disease. In this study, we focused on aortic microcalcification in MFS, but our findings might also apply to other aortopathies. Patients with bicuspid aortic valves, patients with familial thoracic aortic aneurysm and dissection or chronic heart failure patients with left ventricular assist devices also show aortic elastinolysis [69-71]. The presence of aortic microcalcification and its role in these aortopathies, however, still need to be determined.

Visualization of microcalcification in vivo in humans is feasible with positron emission tomography and X-ray $\mathrm{CT}$ imaging with $\left[{ }^{18} \mathrm{~F}\right]$ sodium fluoride as tracer, which adsorbs to calcified deposits [72]. So far, aortic calcification has been underappreciated in aortopathies, but we now demonstrate that, especially, aortic microcalcification at sites of elastic lamina fragmentation is abundant and may contribute to aortic destabilization. Our data strongly support the performance of a human study to determine the predictive value of local aortic 
microcalcification, as determined by aortic imaging with the $\left[{ }^{18} \mathrm{~F}\right.$ - $]$ sodium fluoride tracer, as a risk factor for aortic growth or, even more relevant, as an indicator of an increased risk for aortic dissection.

\section{Acknowledgements}

We acknowledge the financial support from the AMC Graduate School (SH: AMC PhD Scholarship), the Netherlands Heart Institute (VdW: ICIN Incentive grant), Swaenenburgh Stichting (VdW), and the Horstingstuit Foundation (VdW).

\section{Author contributions statement}

The authors contributed in the following way: SW, SH, JE, VdW: conceived experiments; SW, SH, YR, CvR, MV, IvdM, NvV, RF, LvR: carried out the experiments; SW, SH, JE, VdW: analysed the data. All authors were involved in writing the manuscript.

\section{References}

1. Judge DP, Dietz HC. Marfan's syndrome. Lancet 2005; 366: 247-254.

2. Rock MJ, Cain SA, Freeman LJ, et al. Molecular basis of elastic fiber formation: critical interactions and a tropoelastin-fibrillin-1 cross-link. J Biol Chem 2004; 279: 23748-23758.

3. Chung AW, Au Yeung K, Sandor GG, et al. Loss of elastic fiber integrity and reduction of vascular smooth muscle contraction resulting from the upregulated activities of matrix metalloproteinase-2 and -9 in the thoracic aortic aneurysm in Marfan syndrome. Circ Res 2007; 101: 512-522.

4. Angelica MD, Fong Y. Doxycycline delays aneurysm rupture in a mouse model of Marfan syndrome. J Vasc Surg 2008; 141: 520-529.

5. Milewicz DM. Treatment of aortic disease in patients with Marfan syndrome. Circulation 2005; 111: e150-e157.

6. Cozijnsen L, Braam RL, Waalewijn RA, et al. What is new in dilatation of the ascending aorta?: review of current literature and practical advice for the cardiologist. Circulation 2011; 123: 924-928.

7. Erbel R, Eggebrecht H. Aortic dimensions and the risk of dissection. Heart 2006; 92: 137-142.

8. Jondeau G, Detaint D, Tubach F, et al. Aortic event rate in the Marfan population: a cohort study. Circulation 2012; 125: 226-232.

9. Legget ME, Unger TA, O'Sullivan CK, et al. Aortic root complications in Marfan's syndrome: identification of a lower risk group. Heart 1996; 75: 389-395.

10. Silverman DI, Gray J, Roman MJ, et al. Family history of severe cardiovascular disease in Marfan syndrome is associated with increased aortic diameter and decreased survival. J Am Coll Cardiol 1995; 26: 1062-1067.

11. Wanga S, Silversides CK, Dore A, et al. Pregnancy and thoracic aortic disease - managing the risks. Can J Cardiol 2015; 32: 78-85.

12. den Hartog AW, Franken R, Zwinderman AH, et al. The risk for type B aortic dissection in Marfan syndrome. J Am Coll Cardiol 2015; 65: 246-254.

13. Blanchevoye $\mathrm{C}$, Floquet $\mathrm{N}$, Scandolera A, et al. Interaction between the elastin peptide VGVAPG and human elastin binding protein. J Biol Chem 2013; 288: 1317-1328.

14. Booms P, Ney A, Barthel F, et al. A fibrillin-1-fragment containing the elastin-binding-protein GxxPG consensus sequence upregulates matrix metalloproteinase-1: biochemical and computational analysis. J Mol Cell Cardiol 2006; 40: 234-246.
15. Maurice P, Blaise S, Gayral S, et al. Elastin fragmentation and atherosclerosis progression: the elastokine concept. Trends Cardiovasc Med 2013; 23: 211-221.

16. Basalyga DM, Simionescu DT, Xiong W, et al. Elastin degradation and calcification in an abdominal aorta injury model: role of matrix metalloproteinases. Circulation 2004; 110: 3480-3487.

17. Pai A, Leaf EM, El-Abbadi M, et al. Elastin degradation and vascular smooth muscle cell phenotype change precede cell loss and arterial medial calcification in a uremic mouse model of chronic kidney disease. Am J Pathol 2011; 178: 764-773.

18. Bailey M, Xiao H, Ogle M, et al. Aluminum chloride pretreatment of elastin inhibits elastolysis by matrix metalloproteinases and leads to inhibition of elastin-oriented calcification. Am J Pathol 2001; 159: $1981-1986$.

19. Khavandgar Z, Roman H, Li J, et al. Elastin haploinsufficiency impedes the progression of arterial calcification in mgp-deficient mice. J Bone Miner Res 2014; 29: 327-337.

20. Wang SS, Martin LJ, Schadt EE, et al. Disruption of the aortic elastic lamina and medial calcification share genetic determinants in mice. Circ Cardiovasc Genet 2009; 2: 573-582.

21. Simionescu A, Philips K, Vyavahare N. Elastin-derived peptides and TGF- $\beta 1$ induce osteogenic responses in smooth muscle cells. Biochem Biophys Res Commun 2005; 334: 524-532.

22. Simionescu A, Simionescu DT, Vyavahare NR. Osteogenic responses in fibroblasts activated by elastin degradation products and transforming growth factor- $\beta 1$. Am J Pathol 2007; 171: $116-123$.

23. Lee JS, Basalyga DM, Simionescu A, et al. Elastin calcification in the rat subdermal model is accompanied by up-regulation of degradative and osteogenic cellular responses. Am J Pathol 2006; 168: 490-498.

24. Bastos Goncalves F, Voute MT, Hoeks SE, et al. Calcification of the abdominal aorta as an independent predictor of cardiovascular events: a meta-analysis. Heart 2012; 98: 988-994.

25. Ladich E, Yahagi K, Romero ME, et al. Vascular diseases: aortitis, aortic aneurysms, and vascular calcification. Cardiovasc Pathol 2016; 25: 432-441.

26. Mizobuchi M, Towler D, Slatopolsky E. Vascular calcification: the killer of patients with chronic kidney disease. J Am Soc Nephrol 2009; 20: $1453-1464$.

27. Buijs RVC, Willems TP, Tio RA, et al. Calcification as a risk factor for rupture of abdominal aortic aneurysm. Eur J Vasc Endovasc Surg 2013; 46: 542-548.

28. Demer LL, Tintut Y. Vascular calcification: pathobiology of a multifaceted disease. Circulation 2008; 117: 2938-2948.

29. Doherty TM, Asotra K, Fitzpatrick LA, et al. Calcification in atherosclerosis: bone biology and chronic inflammation at the arterial crossroads. Proc Natl Acad Sci U S A 2003; 100: 11201-11206.

30. Pugliese G, Iacobini C, Fantauzzi CB, et al. The dark and bright side of atherosclerotic calcification. Atherosclerosis 2015; 238: 220-230.

31. Ehara S, Kobayashi Y, Yoshiyama M, et al. Spotty calcification typifies the culprit plaque in patients with acute myocardial infarction: an intravascular ultrasound study. Circulation 2004; 110: 3424-3429.

32. Chen W, Dilsizian V. Targeted PET/CT imaging of vulnerable atherosclerotic plaques: microcalcification with sodium fluoride and inflammation with fluorodeoxyglucose. Curr Cardiol Rep 2013; 15 : 364.

33. New SEP, Goettsch C, Aikawa M, et al. Macrophage-derived matrix vesicles: an alternative novel mechanism for microcalcification in atherosclerotic plaques. Circ Res 2013; 113: 72-77.

34. Ewence AE, Bootman M, Roderick HL, et al. Calcium phosphate crystals induce cell death in human vascular smooth muscle cells: a potential mechanism in atherosclerotic plaque destabilization. Circ Res 2008; 103: 28-35. 
35. Kelly-Arnold A, Maldonado N, Laudier D, et al. Revised microcalcification hypothesis for fibrous cap rupture in human coronary arteries. Proc Natl Acad Sci U S A 2013; 110: 10741-10746.

36. Vengrenyuk Y, Carlier S, Xanthos S, et al. A hypothesis for vulnerable plaque rupture due to stress-induced debonding around cellular microcalcifications in thin fibrous caps. Proc Natl Acad Sci U S A 2006; 103: 14678-14683.

37. Dale MA, Xiong W, Carson JS, et al. Elastin-derived peptides promote abdominal aortic aneurysm formation by modulating M1/M2 macrophage polarization. J Immunol 2016; 196: 4536-4543.

38. Pereira L, Lee SY, Gayraud B, et al. Pathogenetic sequence for aneurysm revealed in mice underexpressing fibrillin-1. Proc Natl Acad Sci U S A 1999; 96: 3819-3823.

39. Hanada K, Vermeij M, Garinis GA, et al. Perturbations of vascular homeostasis and aortic valve abnormalities in fibulin-4 deficient mice. Circ Res 2007; 100: 738-746.

40. Guo G, Muñoz-garcía B, Ott CE, et al. Antagonism of GxxPG fragments ameliorates manifestations of aortic disease in Marfan syndrome mice. Hum Mol Genet 2013; 22: 433-443.

41. Franken R, Hibender S, den Hartog AW, et al. No beneficial effect of general and specific anti-inflammatory therapies on aortic dilatation in Marfan mice. PLoS One 2014; 9: e107221.

42. Groenendijk BCW, Benus GFJD, Klous A, et al. Activin A induces a non-fibrotic phenotype in smooth muscle cells in contrast to TGF- $\beta$. Exp Cell Res 2011; 317: 131-142.

43. Geisterfer AA, Peach MJ, Owens GK. Angiotensin II induces hypertrophy, not hyperplasia, of cultured rat aortic smooth muscle cells. Circ Res 1988; 62: 749-756.

44. Petersen E, Wågberg F, Ängquist KA. Serum concentrations of elastin-derived peptides in patients with specific manifestations of atherosclerotic disease. Eur J Vasc Endovasc Surg 2002; 24: $440-444$.

45. Floquet N, Héry-Huynh S, Dauchez M, et al. Structural characterization of VGVAPG, an elastin-derived peptide. Biopolym Pept Sci Sect 2004; 76: 266-280.

46. Golub EE, Boesze-Battaglia K. The role of alkaline phosphatase in mineralization. Curr Opin Orthop 2007; 18: 444-448.

47. Narisawa S, Harmey D, Yadav MC, et al. Novel inhibitors of alkaline phosphatase suppress vascular smooth muscle cell calcification. $J$ Bone Miner Res 2007; 22: 1700-1710.

48. Roy ME, Nishimoto SK. Matrix Gla protein binding to hydroxyapatite is dependent on the ionic environment: calcium enhances binding affinity but phosphate and magnesium decrease affinity. Bone 2002; 31: 296-302.

49. Yao Y, Zebboudj AF, Shao E, et al. Regulation of bone morphogenetic protein-4 by matrix GLA protein in vascular endothelial cells involves activin-like kinase receptor 1. J Biol Chem 2006; 281: 33921-33930.

50. Bunton TE, Biery NJ, Myers L, et al. Phenotypic alteration of vascular smooth muscle cells precedes elastolysis in a mouse model of Marfan syndrome. Circ Res 2001; 88: 37-43.

51. Shao JS, Sierra OL, Cohen R, et al. Vascular calcification and aortic fibrosis: a bifunctional role for osteopontin in diabetic arteriosclerosis. Arterioscler Thromb Vasc Biol 2011; 31: 1821-1833.

52. Aikawa E, Nahrendorf M, Figueiredo JL, et al. Osteogenesis associates with inflammation in early-stage atherosclerosis evaluated by molecular imaging in vivo. Circulation 2007; 116: 2841-2850.

53. Ruiz JL, Hutcheson JD, Aikawa E. Cardiovascular calcification: current controversies and novel concepts. Cardiovasc Pathol 2015; 24: $207-212$.

54. Holm TM, Habashi JP, Doyle JJ, et al. Noncanonical TGFb signaling contributes to aortic aneurysm progression in Marfan syndrome mice. Science 80 2011; 332: 358-361.
55. Habashi JP, Doyle JJ, Holm TM, et al. Angiotensin II type 2 receptor signaling attenuates aortic aneurysm in mice through ERK antagonism. Science 80 2011; 332: 361-365.

56. Cook JR, Clayton NP, Carta L, et al. Dimorphic effects of transforming growth factor signaling during aortic aneurysm progression in mice suggest a combinatorial therapy for Marfan syndrome. Arterioscler Thromb Vasc Biol 2015; 35: 911-917.

57. Nollen GJ, Groenink M, Tijssen JGP, et al. Aortic stiffness and diameter predict progressive aortic dilatation in patients with Marfan syndrome. Eur Heart J 2004; 25: 1146-1152.

58. Luo G, Ducy P, McKee MD, et al. Spontaneous calcification of arteries and cartilage in mice lacking matrix GLA protein. Nature 1997; 386: 78-81.

59. Malhotra R, Burke MF, Martyn T, et al. Inhibition of bone morphogenetic protein signal transduction prevents the medial vascular calcification associated with matrix gla protein deficiency. PLoS One 2015; 10: $1-21$.

60. Duca L, Debelle L, Debret R, et al. The elastin peptides-mediated induction of pro-collagenase-1 production by human fibroblasts involves activation of MEK/ERK pathway via PKA- and PI3K-dependent signaling. FEBS Lett 2002; 524: 193-198.

61. Xiong W, Meisinger T, Knispel R, et al. MMP-2 regulates Erk1/2 phosphorylation and aortic dilatation in Marfan syndrome. Circ Res 2012; 110: e92-e101.

62. Franceschi RT, Xiao G. Regulation of the osteoblast-specific transcription factor, Runx2: responsiveness to multiple signal transduction pathways. J Cell Biochem 2003; 88: 446-454.

63. Ding HT, Wang CG, Zhang TL, et al. Fibronectin enhances in vitro vascular calcification by promoting osteoblastic differentiation of vascular smooth muscle cells via ERK pathway. $J$ Cell Biochem 2006; 99: 1343-1352.

64. Wagenseil JE, Mecham RP. New insights into elastic fiber assembly. Birth Defects Res C 2007; 81: 229-240.

65. Krohn JB, Hutcheson JD, Martínez-Martínez E, et al. Extracellular vesicles in cardiovascular calcification: expanding current paradigms. J Physiol 2016; 594: 2895-2903.

66. Krohn JB, Hutcheson JD, Martínez-Martínez E, et al. Discoidin domain receptor-1 regulates calcific extracellular vesicle release in vascular smooth muscle cell fibrocalcific response via transforming growth factor- $\beta$ signaling. Arterioscler Thromb Vasc Biol 2016; 36: $525-533$.

67. Chen W, Dilsizian V. Targeted PET/CT imaging of vulnerable atherosclerotic plaques: microcalcification with sodium fluoride and inflammation with fluorodeoxyglucose. Curr Cardiol Rep 2013; 15: $1-6$.

68. Yamanouchi D, Morgan S, Stair C, et al. Accelerated aneurysmal dilation associated with apoptosis and inflammation in a newly developed calcium phosphate rodent abdominal aortic aneurysm model. J Vasc Surg 2012; 56: 455-461.

69. Ambardekar AV, Hunter KS, Babu AN, et al. Changes in aortic wall structure, composition, and stiffness with continuous-flow left ventricular assist devices: a pilot study. Circ Hear Fail 2015; 8: 944-952.

70. Padang R, Bannon PG, Jeremy R, et al. The genetic and molecular basis of bicuspid aortic valve associated thoracic aortopathy: a link to phenotype heterogeneity. Ann Cardiothorac Surg 2013; 2: $83-91$.

71. Milewicz DM, Østergaard JR, Ala-Kokko LM, et al. De novo ACTA2 mutation causes a novel syndrome of multisystemic smooth muscle dysfunction. Am J Med Genet A 2010; 152A: 2437-2443.

72. Irkle A, Vesey AT, Lewis DY, et al. Identifying active vascular microcalcification by (18)F-sodium fluoride positron emission tomography. Nat Commun 2015; 6: 7495. 
SUPPLEMENTARY MATERIAL ONLINE

Supplementary figure legends

Figure S1. The elastin receptor complex is expressed in human and murine smooth muscle cells

Figure S2. OsteoSense-800 and MMPSense-680 in vivo in WT and MFS mice

Figure S3. Elastin breaks in wildtype and Marfan mouse ascending aorta

Table S1. Primers used to detect human and/or mouse mRNAs by RT-qPCR

\section{Years ago in the Journal of Pathology...}

The pathology of the adrenal gland in Cushing's syndrome

A. M. Neville and T. Symington

Regeneration of crushed mammalian skeletal muscle and effects of steroids

J. C. Sloper and G. D. Pegrum

Ultrastructure of globule leucocytes in immune rats infected with Nippostrongylus brasiliensis and their possible relationship to the russell body cell

P. Whur and H. S. Johnston

A delayed prolonged increase in venular permeability following intrapleural injections in the rat

J. V. Hurley and G. B. Ryan

To view these articles, and more, please visit:

www.thejournalofpathology.com

Click 'ALL ISSUES (1892 - 2017)', to read articles going right back to Volume 1, Issue 1.

The Journal of Pathology Understanding Disease
A Journal of

The Pathological Society 